\title{
Nanoelectromechanical tuning of dual-mode resonant metasurfaces
}

\author{
Tianzhe Zheng ${ }^{1}$, Hyounghan Kwon ${ }^{1,2}$, Andrei Faraon ${ }^{1,2, *}$ \\ ${ }^{1}$ T. J. Watson Laboratory of Applied Physics and Kavli Nanoscience Institute, California Institute of Technology, \\ 1200 E. California Blvd., Pasadena, CA 91125, USA \\ ${ }^{2}$ Department of Electrical Engineering, California Institute of Technology, 1200 E. California Blvd., Pasadena, \\ CA 91125, USA \\ *Corresponding author: faraon@caltech.edu
}

\begin{abstract}
We demonstrate nanoelectromechanical tuning of dual-mode resonant dielectric metasurfaces. The devices achieve intensity modulation over $40 \%,>10 \mathrm{kHz}$ speed, and $144^{\circ}$ phase shift with 7V bias voltage. (C) 2021 The Author(s)
\end{abstract}

\section{Introduction}

Recently, tunable metasurfaces attracted great interest because they open the path towards manipulating light in time domain. To enhance the tunability of metasurfaces, it is preferred to operate them near resonance and make them sensitive to external perturbations. Dielectric metasurfaces are known to host various free-space coupled resonances including guided mode resonance (GMR) [1] and bound state in the continuum (BIC) modes [2]. In the last few years, the BIC modes or quasi-BIC modes attracted a lot of attentions because of intrinsic higher quality factor and a path to control the strength of the radiation channel through symmetry-breaking perturbation [3]. Nanoelectromechanical systems (NEMS) provide a promising active platform for realizing tunable metasurfaces as they offer advantages in terms of large tunability, low power consumption and the possibility for integration with electronic circuits [4]. In this work, we demonstrate NEMS-tunable dual-mode resonant dielectric metasurfaces hosting GMR and BIC modes. The devices achieve reconfigurable spectral shifts, large reflection, and phase modulation with a speed of over $10 \mathrm{kHz}$ in air.

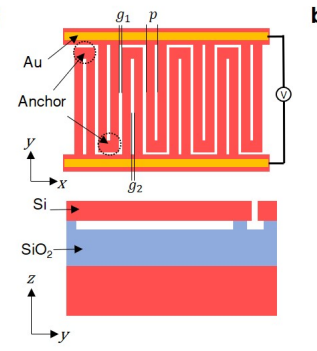

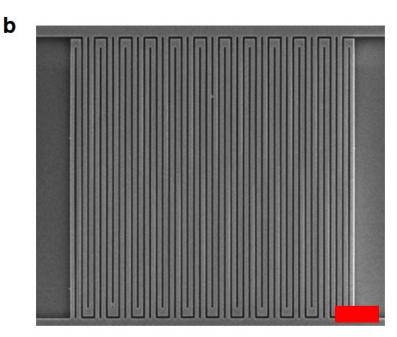

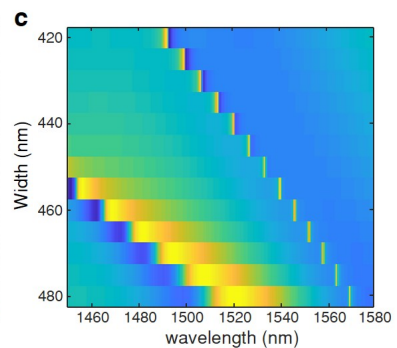

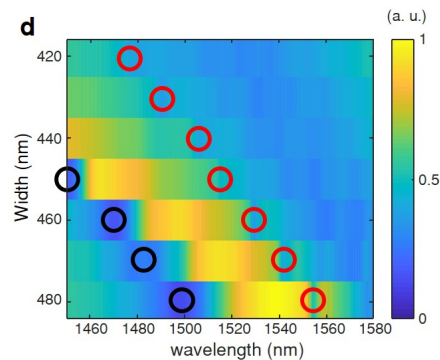

Fig. 1: (a) Top and side view of the device. (b) SEM image of the gratings. The scale bar denotes $5 \mathrm{um}$. (c) Calculated reflection spectra (TE-polarized light) for gratings. (d) Measured reflection of TE-polarized light for the fabricated gratings with $6^{\circ}$ tilted incident light. Black and red circles represent positions of GMRs and quasiBIC modes, respectively.

\section{Results and Discussion}

Top and side views of the design is showed in Figure 1(a).The device contains two sets of suspended doped Si gratings placed interdigitally. Every two bars within the same set are linked to the same Au electrode. $g_{1}\left(g_{2}\right)$ shown in Figure 1(a) is the gap between the nanobars having different (same) voltages. All structures presented here are based on arrays of $500 \mathrm{~nm}$ thick and $30 \mu \mathrm{m}$ long silicon bars. To avoid unwanted diffraction, we ensure that the period of a pair of nanobars $(2 p)$ is smaller than the operating wavelength range. The scanning electron image of the fabricated device is in Figure 1(b). The metasurface is designed to host GMR and quasi-BIC modes in the telecom wavelength range. Calculated and measured spectra of reflection are plotted in Figure 1(c) and (d), showing the two resonant modes and good agreement.

The reconfigurabiility is demonstrated in Figure 2. When a voltage is added on the electrodes, the bars connected to different ends will attract each other. $g_{1}\left(g_{2}\right)$ will shrink (expand) continuously as a function of external bias. 


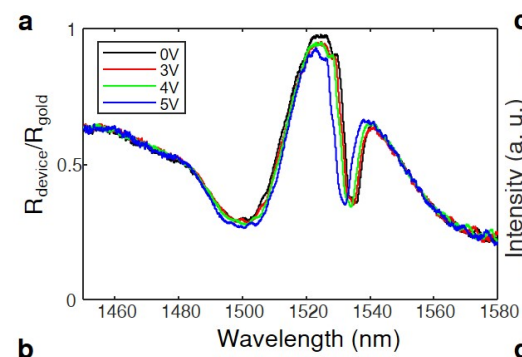

b

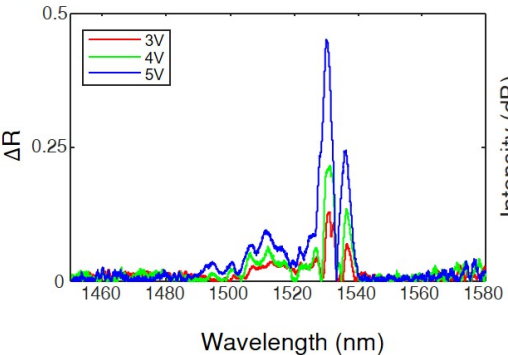

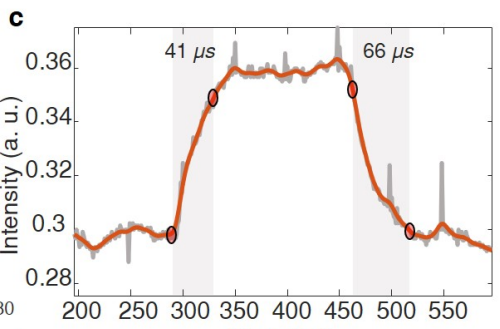

d

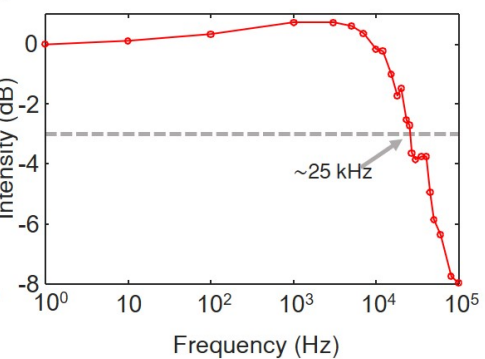

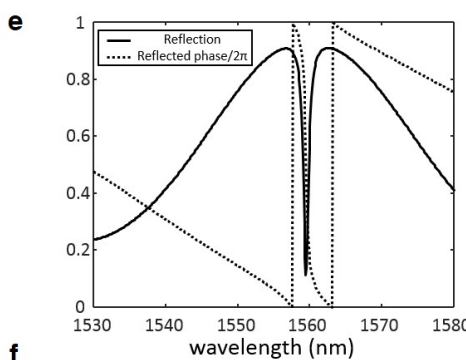

$\mathbf{f}$

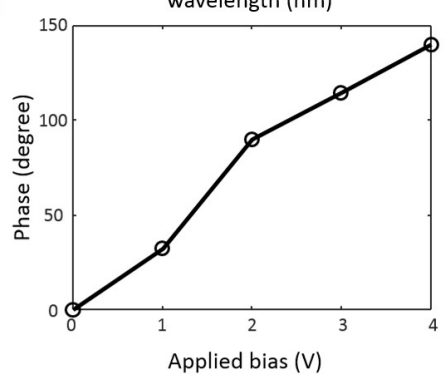

Fig. 2: (a) Measured reflection spectra of TE-polarized lights under different voltages. (b) Spectra of absolute modulation in reflection calculated from (a). (c) Measured timing response in a period of $3 \mathrm{kHz}$ square-wave signal whose duty cycle and amplitude are 0.5 and 6V. (d) Measured frequency response of the grating. Cutoff frequency is $\sim 25 \mathrm{kHz}$. (e) Calculated reflection and phase response when two mode overlaps. (f) Measured phase response under different applied bias.

Thus, the position of GMR and quasi-BIC will be controlled continuously by the bias voltage. First, the reflection response of the device under static external bias is performed. In Figure 2(a), the BIC mode shows blueshift up to $5 \mathrm{~nm}$ when increasing the voltages up to $5 \mathrm{~V}$. The absolute intensity change is plotted in Figure 2(b) as the function of wavelength. The maximum absolute change of intensity is up to 0.45 around the quasi-BIC resonance.

Figure 2(c) and (d) shows the the temporal response of our NEMS device in the air. A $3 \mathrm{kHz}$ square-wave signal is applied to the electrodes on the device used in Figure 2(b). The measured output signal is plotted in Figure 2(c). The measured rise time (up to 90\% power) and fall times (down to 10\% power) shown in Figure 2(c) are 41 and 66 $\mu \mathrm{s}$, respectively. The rise and fall times indicate the speed limit of $15.2 \mathrm{kHz}$. The frequency response is measured and plotted in Figure 2(d) showing the cutoff frequency of $25 \mathrm{kHz}$. The frequency response is currently limited by air damping. Since the mechanical frequency of the nanobar is around $4.5 \mathrm{MHz}$, megahertz speeds are expected for a device packaged in vacuum.

Finally, we demonstrate enhanced phase response through interference between the GMR and quasi-BIC mode. When the GMR and BIC resonances overlap, the interference of two modes could enable large phase shifts with non-zero reflection. Figure 2(e) shows the numerical result of the reflection phase and amplitude when the quasiBIC mode is placed near the peak of GMR. A $2 \pi$ phase change occurs along with the quasi-BIC resonance, which indicates the phase enhancement due to interference between modes. In experiment, we observe the similar effect in Figure 2(f). A relative phase shift of $144^{\circ}$ is achieved at $1566 \mathrm{~nm}$ under different bias voltages. The deviation from the simulation is primarily due to limited free-space coupling to the quasi-BIC mode.

In conclusion, we demonstrate NEMS tuning of the GMR and BIC doubly-resonant metasurfaces with fast and large modulation both in intensity and phase. We envision that this will pave the way towards NEMS tunable metasurfaces with more applications, such as spatial light modulators, lasers, and pulse controllers.

\section{References}

1. G. Quaranta, G. Basset, O. J. Martin, and B. Gallinet, "Recent advances in resonant waveguide gratings," Laser \& Photonics Rev. 12, 1800017 (2018).

2. C. W. Hsu, B. Zhen, A. D. Stone, J. D. Joannopoulos, and M. Soljačić, "Bound states in the continuum," Nat. Rev. Mater. 1, 1-13 (2016).

3. K. Koshelev, S. Lepeshov, M. Liu, A. Bogdanov, and Y. Kivshar, "Asymmetric metasurfaces with high-q resonances governed by bound states in the continuum," Phys. review letters 121, 193903 (2018).

4. N. I. Zheludev and E. Plum, "Reconfigurable nanomechanical photonic metamaterials," Nat. nanotechnology 11, 16 (2016). 